\title{
Estrategia de contratación de personal. Una experiencia en la industria
}

\author{
Personnel recruitment strategy. an experience in the industry
}

Estratégia de recrutamento de pessoal. uma experiência na indústria

\author{
Recibido: enero 2020 \\ Arbitrado: febrero 2020 \\ Publicado: mayo 2020
}

《richelle Perozo

michelle_cpc@hotmail.com ORCID: 0000-0003-4815-2719

Universidad Nacional Experimental "Rafael María Baralt". Venezuela
RESUMEN

El requerimiento de destrezas, capacidades o competencias del saber hacer se convierte en uno de los activos empresariales más importantes. En este sentido, determinar la estrategia de contratación de personal que se aplica en la industria petrolera venezolana es el propósito de esta investigación de tipo descriptiva, con diseño no experimental, transeccional y de campo. Para ello se utilizó la información suministrada por los gerentes de las tres (3) empresas activas prestatarias del servicio de perforación de pozos a la industria petrolera venezolana. Los resultados permiten afirmar que el llevar a cabo una estrategia de contratación exitosa involucra inferir el desempeño de un candidato ante un puesto de trabajo específico, considerando su proceso continuo de aprendizaje y desarrollo en términos de autosuficiencia y adaptación a nuevos escenarios, sin que su formación previa afecte.

Palabras clave: Capacidades; contratación de personal; desempeño, estrategia, industria petrolera venezolana
ABSTRACT

RESUMO
The requirement of skills, capacities or competencies of know-how becomes one of the most important business assets. In this sense, determining the personnel hiring strategy applied in the Venezuelan oil industry is the purpose of this descriptive research, with a nonexperimental, transectional and field design. For this, the information provided by the managers of the three (3) active companies that provided well drilling services to the Venezuelan oil industry was used. The results allow us to affirm that carrying out a successful hiring strategy involves inferring the performance of a candidate in a specific job position, considering their continuous learning and development process in terms of selfsufficiency and adaptation to new scenarios, without their training prior affect.

Key words: Capabilities; personnel hiring; performance; strategy; Venezuelan oil industry um dos ativos mais importantes do negócio. Nesse sentido, determinar a estratégia de contratação de pessoal aplicada na indústria do petróleo venezuelana é o objetivo desta pesquisa descritiva, com um desenho não experimental, transversal e de campo. Para tanto, foram utilizadas as informações fornecidas pelos gestores das três (3) empresas ativas que prestam serviços de perfuração de poços à indústria petrolífera venezuelana. Os resultados permitem afirmar que realizar uma estratégia de contratação bem-sucedida envolve inferir o desempenho de um candidato em determinado cargo, considerando seu processo contínuo de aprendizagem e desenvolvimento em termos de autossuficiência e adaptação a novos cenários, sem que sua formação afete prévia.

Palavras chave: Capacidades; recrutamento; desempenho, estratégia, indústria de petróleo venezuelana 


\section{INTRODUCCIÓN}

$\mathrm{E}$ n la actualidad, las empresas se encuentran con un mercado altamente competitivo, globalizado, con una nueva visión para hacer negocios, con una tecnología que avanza vertiginosamente y una brecha significativa entre sus competidores, sumado a ello, un entorno macro-económico de gran incertidumbre. Lo determinante ante esta realidad, es reaccionar, pero de forma inmediata, para detectar la brecha y poder re-direccionar la estrategia, o formularla en virtud de desarrollar competencias para identificar los factores claves de éxito del negocio.

Desde esta perspectiva, las empresas necesitan estar a la vanguardia en conocimiento de herramientas gerenciales, que les permitan mejorar su competitividad y desempeño basado en la toma de decisiones. Dentro de este contexto, es importante determinar si las estrategias formuladas están satisfaciendo expectativas de rendimiento; su enfoque plantea que las empresas no deberían medir los resultados sólo en términos de utilidad o pérdida, tal como lo esbozan los modelos de gestión tradicionales, siendo necesario incluir el análisis del personal calificado.

En esta perspectiva el tratamiento del recurso humano cambia su paradigma, modificándose las formas de contratación, recurriendo a los contratos atípicos. Bajo esta premisa se analiza, desde el punto de vista teórico, las estrategias de contratación de personal en las empresas. Es así, como cada vez más existen mayor cantidad de estudios en empresas en todo el mundo que destacan la influencia de las personas en la rentabilidad de las empresas, pues el recurso humano es un factor significativo en la toma de decisiones estratégicas, y es el que se encarga de dirigir y ejecutar las operaciones de la organización (Bossidy y Charan, 2017).

Ante esta realidad, muchas empresas están acudiendo a la externalización, disminuyendo la verticalidad de las organizaciones a través de la subcontratación de servicios, deslastrarse de etapas no claves o costosas del proceso productivo. Se trata por lo tanto, seguir posicionados en el mercado con una alta competitividad, pero fuera del modelo económico tradicional que ya no les resulta rentable.

En el caso venezolano, específicamente en el sector petrolero, se ubican las empresas prestatarias del servicio de perforación de pozos a la industria petrolera venezolana. Este servicio se realiza mediante contratos entre estas empresas y PDVSA, donde se establecen cláusulas y especificaciones técnicas requeridas para el servicio a prestar. En este sentido, se requiere de acuerdo a Duran (2013), una mano de obra calificada que permita reducir costos y perfeccionar la calidad.

La competitividad de las empresas se relaciona con la capacidad de innovación y creatividad del recurso humano, siendo este el responsable de alcanzar la eficiencia y productividad que necesitan las organizaciones para posicionarse en el mercado mundial. 
Ante este contexto, este trabajo tiene como propósito determinar la estrategia de contratación de personal que se aplica en la industria petrolera venezolana, destacando el reclutamiento del personal como estrategia empresarial, la selección del personal, así como la compensación.

Estas estrategias deben ser coherentes no solo con el área operativa, sino también con los objetivos organizacionales, es por ello que tienen tanta importancia, pues acarrean consecuencias para toda la organización y son el origen de las políticas que se generan en la empresa, el punto de partida de todo el resto de la planificación.

\section{MÉTODO}

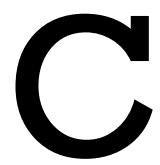

onsiderando el problema planteado y los objetivos a alcanzar, el tipo de investigación se catalogó como descriptiva, pues persiguió describir sistemáticamente, características homogéneas de los fenómenos estudiados sobre la realidad (individuos, comunidades). Los estudios descriptivos buscan especificar características y perfiles de personas, grupos, comunidades, procesos, objetos o cualquier otro fenómeno que se someta a un análisis (Hernández y otros. 2014). Al respecto, de acuerdo a Abreu (2016), los estudios descriptivos se centran en recoger información relacionada con el estado real de las personas, objetos, situaciones o fenómenos.

Asimismo, el diseño seleccionado, según el tipo de investigación realizada, fue el no experimental pues, según estos autores, se realiza sin manipular la variable intencionalmente, a su vez, se observa el fenómeno tal y como se presente en su contexto natural para después analizarlo; este tipo de diseño es más cercano a la realidad estudiada y posee un control menos riguroso que el experimental.

De igual manera, se clasificó con diseño transeccional, dado que la variable estrategias de contratación de personal se estudió en un sólo momento del tiempo, octubre de 2020, y se midió según los criterios de la población que proporcionó la data necesaria para el estudio. Finalmente, se calificó de campo, pues lo datos se recogieron en forma directa de la realidad, además, la información fue recabada a través del cuestionario, realizando preguntas pertinentes a los objetivos de la investigación

Los datos se recolectaron de una población finita, conformada por las empresas prestatarias del servicio de perforación de pozos a la industria petrolera venezolana en el Lago de Maracaibo, donde se ubicaron tres (3) empresas activas para el momento de la investigación. Los sujetos informantes fueron directamente el personal calificado de gerentes generales, gerentes de perforación y gerentes de mantenimiento, representada por un total de 9 personas.

La técnica de recolección de datos fue la encuesta, y el instrumento un cuestionario con 9 reactivos bajo una escala tipo Lickert, representada de la siguiente forma: Totalmente de acuerdo (TA), De acuerdo (DA), Ni de acuerdo ni en desacuerdo (NI), En desacuerdo (ED), y Totalmente en 
desacuerdo (TD). Se utilizó el juicio de expertos para la validez, y el coeficiente Alfa de Cronbach para su confiabilidad, arrojando un valor de 0,872 considerado de muy alta confiabilidad. Se aplicó como técnica para el análisis de datos el análisis estadístico descriptivo, con el cálculo de la media aritmética $(\overline{\mathrm{X}})$, bajo la interpretación del baremo correspondiente.

\section{RESULTADOS}

$\mathrm{L}$ os valores presentados en la tabla 1, corresponden al indicador reclutamiento del personal. En los mismos se aprecia como la media se ubicó en 3,96 indicando la existencia de alta aplicación de las actividades y procesos asociados al mismo, es decir, en las empresas bajo estudio el reclutamiento del personal como parte de la estrategia de contratación de personal se aplica de manera alta.

Tal resultado obedece al nivel de aplicación otorgado a cada ítem asociado al indicador, donde otorgaron muy alta aplicación a si al momento de requerir personal para un cargo vacante se le da oportunidad al personal interno (ascensos, traslados, promociones) $(4,44)$, y poseen programas de desarrollo de personal $(4,44)$, consideradas fortalezas de sus estrategias de contratación de personal.

Tabla 1. Reclutamiento del personal.

\begin{tabular}{|c|c|c|}
\hline \multicolumn{3}{|c|}{$\begin{array}{l}\text { Variable: Estrategias de operaciones } \\
\text { Dimensión: Estrategias de contratación de personal }\end{array}$} \\
\hline ÍTEM & & \\
\hline En la empresa donde usted labora: & $\overline{\boldsymbol{X}}$ & CATEGORÍA \\
\hline $\begin{array}{l}\text { 1. Al momento de requerir personal para un cargo vacante se le } \\
\text { da oportunidad al personal interno (ascensos, traslados, } \\
\text { promociones). }\end{array}$ & 4,44 & $\begin{array}{l}\text { Muy alta aplicación } \\
\text { Fortaleza }\end{array}$ \\
\hline 2. Poseen programas de desarrollo de personal. & 4,44 & $\begin{array}{l}\text { Muy alta aplicación } \\
\text { Fortaleza }\end{array}$ \\
\hline $\begin{array}{l}\text { 3. Tiene asociaciones con instituciones externas para el } \\
\text { reclutamiento de personal (universidades, centros de } \\
\text { capacitación u otros centros gremiales). }\end{array}$ & 3,00 & $\begin{array}{l}\text { Moderada aplicación } \\
\text { Debilidad }\end{array}$ \\
\hline Total indicador: & 3,96 & $\begin{array}{l}\text { Alta aplicación } \\
\text { Fortaleza }\end{array}$ \\
\hline
\end{tabular}

Sin embargo, otorgan moderada aplicación en cuanto a si tienen asociaciones con instituciones externas para el reclutamiento de personal (universidades, centros de capacitación u otros centros gremiales) $(3,00)$, vista como debilidad de sus estrategias. 
De acuerdo a estos resultados, se valida lo indicado por Verdejo (2014) cuando afirma, el ingreso de nuevos elementos a la empresa trae consigo ventajas en la importación de ideas nuevas y diferentes enfoques acerca de los problemas internos; también, renueva así como enriquece los recursos humanos y aprovecha las inversiones en preparación y desarrollo del personal efectuadas por otras empresas o por ellos mismos. Igualmente, acudir al reclutamiento externo, generalmente, absorbe más tiempo que el interno pues requiere la utilización de apropiadas técnicas de selección y el uso efectivo de fuentes que permitan la captación de personal.

De igual forma, se logra validar la posición de la investigadora cuando señala, ambas fuentes deben ser factibles de aplicarse en la práctica de las empresas prestatarias del servicio de perforación de pozos a la industria petrolera venezolana en el lago de Maracaibo, pues se puede presentar la oportunidad de promocionar a los trabajadores internos o la necesidad de captar personal externo en la organización.

De seguido, en la tabla 2, se muestra la información correspondiente al indicador selección del personal; según estos datos, se aprecia para el indicador una media de 4,00 indicando alta aplicación de la selección del personal como parte de la estrategia de contratación de personal aplicada en las empresas bajo estudio.

Tabla 2. Selección del personal.

\section{Variable: Estrategias de operaciones Dimensión: Estrategias de contratación de personal ÍTEM}

En la empresa donde usted labora:

4. Realizan comparación entre los requisitos del cargo y el perfil de candidatos al momento de la selección del personal.

3,89

3,78

4,33

Muy alta aplicación candidatos.

$\overline{\boldsymbol{X}}$

CATEGORÍA

5. Tienen entrevistas preestablecidas para la selección del personal.

6. Realizan pruebas de conocimientos o capacidad de estudio a los

Fortaleza

\begin{tabular}{ccc}
\hline Total indicador: & 4,00 & $\begin{array}{c}\text { Alta aplicación } \\
\text { Fortaleza }\end{array}$ \\
\hline
\end{tabular}

De tal forma, se aplica de manera muy alta el que realicen pruebas de conocimientos o capacidad de estudio a los candidatos $(4,33)$; a su vez, catalogan de forma alta, el que realicen comparación entre los requisitos del cargo y el perfil de candidatos al momento de la selección del personal $(3,89)$; y si tienen entrevistas preestablecidas para la selección del personal $(4,11)$; siendo todos estos aspectos fortalezas de su selección del personal, como parte de sus estrategias de contratación. 
Con base a estos resultados, se valida lo indicado por Invancevich y otros. (2010) cuando aseguran, la selección de personal busca solucionar dos (2) problemas claves, como: adecuación del personal al cargo o eficiencia del personal al cargo. En general, puede decirse que el proceso selectivo debe analizarse como una comparación entre los requisitos del cargo y el perfil de los candidatos que se presentan, la cual se obtiene por medio de diferentes técnicas de selección, a través de un proceso de toma de decisión, lo cual representa la parte fundamental del proceso ya que se rechaza o se acepta a los aspirantes a ocupar el cargo vacante.

De manera similar, también se logra coincidencia con lo expuesto por la investigadora cuando afirma, el proceso de selección, como fase inmediatamente siguiente al proceso de reclutamiento, es donde la empresa debe seleccionar de lista de solicitantes al personal que cumpla mejor con los criterios para ocupar el puesto vacante, la misma debe realizarse con base a los aspectos del trabajo a desempeñar, se deben realizar exámenes psicotécnicos, y evaluar las habilidades y competencias de los candidatos.

Con relación al último indicador de esta dimensión denominado compensación, la tabla 3 muestra la información recabada para el mismo. Se observa una media de 3,48, otorgando alta aplicación de las actividades asociadas a este indicador en las empresas prestatarias del servicio de perforación de pozos a la industria petrolera venezolana en el lago de Maracaibo; lo cual refleja que, la compensación como parte de la estrategia de contratación de personal aplicada en las empresas bajo estudio ostenta alta aplicación, siendo considerada una fortaleza, según el baremo de interpretación de resultados establecido.

Tabla 3. Indicador: Compensación.

\begin{tabular}{|c|c|c|}
\hline \multicolumn{3}{|c|}{$\begin{array}{l}\text { Variable: Estrategias de operaciones } \\
\text { Dimensión: Estrategias de contratación de personal }\end{array}$} \\
\hline $\begin{array}{c}\text { ÍTEM } \\
\text { En la empresa donde usted labora: }\end{array}$ & $\overline{\boldsymbol{X}}$ & CATEGORÍA \\
\hline $\begin{array}{l}\text { 7. La remuneración salarial es similar a otras empresas del } \\
\text { sector. }\end{array}$ & 3,44 & $\begin{array}{l}\text { Alta aplicación } \\
\text { Fortaleza }\end{array}$ \\
\hline 8. Se rigen por políticas del gobierno para definir sus salarios. & 3,22 & $\begin{array}{l}\text { Moderada aplicación } \\
\text { Debilidad }\end{array}$ \\
\hline $\begin{array}{l}\text { 9. Utilizan tabuladores para la asignación de salarios en } \\
\text { función de las experiencias del trabajador. }\end{array}$ & 3,78 & $\begin{array}{l}\text { Alta aplicación } \\
\text { Fortaleza }\end{array}$ \\
\hline Total indicador: & 3,48 & $\begin{array}{l}\text { Alta aplicación } \\
\text { Fortaleza }\end{array}$ \\
\hline
\end{tabular}

El valor obtenido, en la media del indicador, es producto de la alta aplicación otorgada a los ítems 43 y 45, al consultarles si la remuneración salarial es similar a otras empresas del sector $(3,44)$; y si utilizan tabuladores para la asignación de salarios en función de las experiencias del 
trabajador $(3,78)$; confiriéndoles fortalezas en estos aspectos. Sin embargo, tachan con moderada aplicación el que se rijan por políticas del gobierno para definir sus salarios $(3,22)$; siendo esto debilidad de su estrategia de compensación.

Corresponde este resultado a lo descrito por Duran (2013) cuando asevera, los salarios deben tener un equilibrio tanto interno como externo. Cuando se habla de lo interno, el salario debe ser equitativo y justo para toda la empresa, y el mismo se logra mediante la evaluación y clasificación de los cargos a través de la política salarial de la misma. Así, las políticas salariales deben contener, estructuras de cargos y salarios, salarios de admisión para las diversas clases salariales y previsión de reajustes salariales. En cuanto al equilibrio externo, se basa en la investigación salarial, de manera que tenga relación con otras empresas similares que actúen en el mercado de trabajo.

También se valida la posición de la investigadora para quien, la remuneración salarial debe ser equitativa tanto dentro de la organización como en comparación con el mercado, con el objetivo de lograr trabajadores conformes y motivados. No obstante, se acota que, en el mercado laboral de Venezuela, los salarios están regidos por tabuladores de sueldos y salarios para los profesionales y no profesionales, tomando en consideración el salario mínimo, el cual es establecido por el Ejecutivo Nacional.

Seguidamente, en la tabla 4, se resume el comportamiento de la estrategia de contratación de personal, con sus respectivos indicadores. En ésta, se aprecia que el valor para la media se ubicó en 3,81 , lo cual indica alta aplicación de la estrategia de contratación de personal en las empresas bajo estudio.

Tabla 4. Estrategia de contratación de personal.

\begin{tabular}{|c|c|c|c|}
\hline INDICADOR & & $\bar{X}$ & CATEGORÍAS \\
\hline Reclutamiento del personal & & 3,96 & $\begin{array}{c}\text { Alta aplicación } \\
\text { Fortaleza }\end{array}$ \\
\hline Selección del personal & & 4,00 & $\begin{array}{l}\text { Alta aplicación } \\
\text { Fortaleza }\end{array}$ \\
\hline Compensación & & 3,48 & $\begin{array}{l}\text { Alta aplicación } \\
\text { Fortaleza }\end{array}$ \\
\hline & RESUMEN DIMENSIÓN & 3,81 & $\begin{array}{c}\text { Alta aplicación } \\
\text { Fortaleza }\end{array}$ \\
\hline
\end{tabular}

Este resultado observado, alta aplicación de la estrategia de contratación de personal, es determinado por el alto nivel de aplicación de cada uno de sus indicadores, puesto de manifiesto en las medias aritméticas alcanzadas: reclutamiento del personal $(3,96)$, selección del personal $(4,00)$, compensación $(3,48)$, evidenciando fortalezas en todos estos aspectos en sus estrategias de contratación de personal. 
Estos resultados validan a Núñez y otros. (2010), cuando aseveran en la actualidad, los planes de acción, de las empresas, están basados en decisiones sobre la fuerza de trabajo, incluyen selección, contratación, capacitación, supervisión, compensación y despido; estas decisiones las toman, con frecuencia, gerentes de línea de operaciones con la asistencia o en forma mancomunada con la gerencia de recursos humanos. Aquí, se acota que administrar la fuerza de trabajo, de manera productiva y humana, es una tarea clave para la función de operaciones, por ello es de suma importancia analizar aspectos estratégicos, tácticos y operativos del personal.

De igual forma, validan la posición de la investigadora cuando afirma, el llevar a cabo una estrategia de contratación exitosa involucra inferir el desempeño de un candidato ante un puesto de trabajo específico, considerando su proceso continuo de aprendizaje y desarrollo en términos de autosuficiencia y adaptación a nuevos escenarios, sin que su formación previa afecte. En consecuencia, hoy día se necesita de procesos de reclutamiento de personal que incluyan evaluaciones de los candidatos centradas en su desempeño y en la adquisición de nuevos conocimientos en diversas condiciones.

\section{CONCLUSIONES}

$\mathbf{S}$ e determinó alta aplicación de la estrategia de contratación de personal en las empresas bajo estudio. Asimismo, se pudo conocer que la mayoría de las actividades medidas en cada tipo de estrategia de contratación de personal arriban a muy alta y alta aplicación, permitiendo concluir que en estas empresas aplican:

- En cuanto al reclutamiento del personal: al momento de requerir personal para un cargo vacante se le da oportunidad al personal interno (ascensos, traslados, promociones); y poseen programas de desarrollo de personal.

- Para la selección del personal: realizan pruebas de conocimientos o capacidad de estudio a los candidatos; realizan comparación entre los requisitos del cargo y perfil de candidatos al momento de la selección del personal; y tienen entrevistas pre establecidas para la selección del personal.

- Por último, en la estrategia compensación: la remuneración salarial es similar a otras empresas del sector; y utilizan tabuladores para la asignación de salarios en función de las experiencias del trabajador. 


\section{REFERENCIAS}

Abreu, J. (2016). Metodología de la investigación: Preguntas. Métodos. Todo menos tesis. Publicaciones Create Space Independent Publishing Platform. Canadá

Bossidy, L.; y Charan, R. (2017). Estrategias operacionales. El arte de la ejecución en los negocios. Editorial Norma. Colombia

Duran, A. (2013). Estrategias de Contratación: Habilidades y Competencias. Blog de reclutamiento 2.0. Disponible en: http://blog.talentclue.com/bid/322802/Estrategi as-de-Contrataci-n-Habilidades-y Competencias Hernández, R.; Fernández, C., y Baptista, M. (2014). Metodología de la investigación. Sexta Edición. Editorial McGraw-Hill. Chile
Invancevich, J.; Konopaske, R.; Matteson M., y Núñez, J. (2010). Comportamiento organizacional y administración de empresas. Décima edición. Editorial McGraw-Hill Interamericana de España S.L. España

Verdejo, S. (2014). Los procesos de reclutamiento y selección como fuente de ventaja competitiva: caso Google Inc. En: https://books.google.co.ve/books?id=0nUzjwEA CAAJ 